BBA 554II

\title{
EFFECT OF CHOLESTEROL INCORPORATION ON THE SENSITIVITY OF LIPOSOMES TO THE POLYENE ANTIBIOTIC, FILIPIN
}

S. C. KINSKY, J. HAXBY, C. B. KINSKY, R. A. DEMEL AND L. L. M. VAN DEENEN

Deparment of Pharmacology, Washington University School of Medicine, St. Louis, Mo. (U.S.A.) and Organisch Chemisch Laboratoritm, Rijksuniversiteit, Utrecht (The Netherlands)

(Received September 29th, 1967)

\section{SUMMARY}

I. A simple spectrophotometric assay has been developed by which the effect of lytic agents on liposomes can be conveniently determined. This method is based on the change in absorbance at $340 \mathrm{~m} \mu$ which occurs when trapped glucose is liberated in the presence of hexokinase (EC 2.7.I.I) and glucose-6-phosphate dehydrogenase (EC I.I.I.49). Because the spectrophotometric assay is more sensitive (requiring lower concentrations of liposomes), it possesses several advantages over alternative procedures which have been used to study the release of trapped markers.

2. It has been reported that the polyene antibiotic, filipin, did not produce a greater release of marker when cholesterol was incorporated into the liposomes. This problem has been re-examined with the spectrophotometric assay. Our results indicate that the presence of sterol does increase the sensitivity of the liposomes to the antibiotic. These observations are consistent with previous lipid monolayer and bilayer experiments and the known biological properties of the polyene antibiotics.

INTRODUCTION

Numerous electron microscopic studies have shown that the predominant structure of aqueous phospholipid dispersions has a lamellar appearance. This pattern has generally been interpreted as an array of concentric bimolecular lipid layers separated from each other by an aqueous compartment. In I965, BANGHAM, STANDISH AND WATKINS ${ }^{1}$ provided additional evidence in favor of this interpretation and demonstrated the feasibility of using these lipid dispersions as model membranes in the study of lytic mechanisms. They were able to show that various marker compounds could be trapped within the aqueous regions and concluded that each bilayer forms an intact membrane which effectively separates these compartments from the external medium in which these lipid dispersions (hereafter referred to as liposomes*) were suspended.

* These have also been referred to as hipid "structures", "spherules" "liquid crystals", "smectic mesophase", and (most appropriately) "Bangasomes" in the literature. We prefer the term liposomes because it is not only simpler but also descriptive of the actual lamellar arrangement of the lipids (i.e., as a "body" containing trapped compounds). 
The effect of the polyene antibiotic, filipin, on lecithin-cholesterol liposomes has been investigated by WEISSMANN AND SESSA ${ }^{2,3}$. They found that filipin released various marker compounds to the same extent from liposomes prepared with and without cholesterol. This observation is difficult to reconcile with the prevailing evidence which indicates that the polyenc antibiotics are selcctively toxic towards those organisms which contain sterol in the cell membrane (reviewed in refs. $4,5,6$ ). It is also at variance with our earlier monolayer and bilayer experiments which have shown that low concentrations of filipin $\left(\mathrm{IO}^{-8}-\mathrm{IO}^{-5} \mathrm{M}\right)$ preferentially interacted with films containing cholesterol ${ }^{7,8}$. We have recently re-examined the effect of the polyenes on single and mixed lipid monolayers and have observed that filipin can increase the surface pressure of lecithin monolayers (in the absence of cholesterol) at high molar ratios of antibiotic/spread lipid ${ }^{9}$. At low molar ratios of filipin/spread lipid, the antibiotic still shows selectivity for monolayers which contain the sterol. As discussed previously ${ }^{9}$, these observations cast some doubt on the significance of the phenomena described by WEISSMANN AND SESSA because of the high antibiotic concentrations ( $\mathrm{IO}^{-4}-\mathrm{IO}^{-3} \mathrm{M}$ ) which were necessary to obtain an appreciable release of marker. In the present study, we have re-examined the action of filipin on liposomes using a sensitive spectrophotometric assay. As demonstrated below, this method may have several advantages over earlier procedures which have been used to determine the effect of the antibiotic on liposomes. Our results indicate that incorporation of cholesterol into liposomes does increase their sensitivity to filipin.

\section{MATERIALS AND METHODS}

\section{Chemicals and enzymes}

These were obtained from the following companies: Boehringer-Mannheim Corporation, New York, N.Y. (hexokinase (EC 2.7.I.I) and glucose-6-phosphate dehydrogenase (EC I.I.I.49); K and K Laboratories, Inc., Plainview, N.Y. (dicetylphosphate); Pierce Chemical Company, Rockford, Ill. (egg lecithin); Rohm and Haas, Philadelphia, Pa. (Triton X-roo); Sigma Chemical Company, St. Louis, Mo. (ATP, TPN, FMN, cholesterol, and tris(hydroxymethyl)aminomethane). Filipin was a generous gift of the Upjohn Company, Kalamazoo, Mich.; we are greatly indebted to Dr. G. B. WhitfiEld for supplying this antibiotic. The lots of filipin used in the present study were more than $90 \%$ pure on the basis of biological activity $(G$. $B$. Whitfield, personal communication)*.

\section{Preparation and assay of liposomes: "macro method"}

The method was modified slightly from that employed by WEISSMANN, SESSA AND WEISSMANN ${ }^{10}$. Chloroform solutions of lecithin (containing 30-90 $\mu$ moles), dicetyl

\footnotetext{
* After completion of this manuscript, we have been informed by Drs. M. E. BERGY and T. E. EBLE (The Upjohn Company, Kalamazoo, Mich.) that they have recently becn able to separate crystalline filipin, which has up to now been regarded as a single chemical entity and for which a structure has been proposed ${ }^{17}$, into 3 major pentaene components. These components are closely related chemically as indicated by elemental analysis, ultraviolet, infrared, and NMR spectra. We have since repeated all the experiments with pure filipin III (which accounts for approximately $50 \%$ of the material present in the filipin complex) with results identical to that reported in this paper.
} 
phosphate, and cholesterol in the desired molar ratio were added to a roo-ml roundbottomed flask and the solvent removed by evaporation under reduced pressure. For every ro $\mu$ moles of lecithin present in the dried lipid film, $1.0 \mathrm{ml}$ of marker solution was added. Depending on the experiment, these solutions were either $0.145 \mathrm{M}$ potassium phosphate $\left(\mathrm{pH}_{4} .9\right)$, or $0.3 \mathrm{M}$ glucose. The lipids were thoroughly agitated with a Vortex mixer, under a $\mathrm{N}_{2}$ atmosphere, until lipid film was no longer detectable on the sides of the flask. The aqueous lipid dispersion was then kept at room temperature (with occasional agitation) for $3-5 \mathrm{~h}$ before subsequent dialysis. In the original procedure described by Bangham, Standish and Watkins', and used by WeissMANN and coworkers, any untrapped marker was removed by dialysis against periodic changes of fresh dialysing medium. In the present study, the liposomes were dialysed overnight (about $I_{5} \mathrm{~h}$ ) at room temperature against $5 \mathrm{l}$ of a solution containing $0.075 \mathrm{M} \mathrm{KCl}$ and $0.075 \mathrm{M} \mathrm{NaCl}$ (hereafter referred to as isotonic salt mixture); the liposome: dialysing solution ratio never exceeded $\mathrm{I}: 560$. Under these conditions, it was routinely observed that the amount of marker released from the dialysed liposome preparation by complete lysis with Triton was al least $3-4$ times greater than the amount present in control tubes (i.e., samples incubated without lytic agent) as determined by the procedure described below.

In the experiments of WEISSMANN, SESSA AND WEISSMANin ${ }^{10}$, the influence of different compounds on the leakage of trapped markers was determined by addition of the compound under investigation directly to the liposomes immediately before transfer to the dialysing sacs. This procedure may have disadvantages when used to study the effect of lytic agents which can pass through the dialysing tubing. In the assay which we have employed, the liposomes were first incubated with the appropriate lytic agent before removal of an aliquot for dialysis. Small test tubes $(70 \mathrm{~mm} x$ roo $\mathrm{mm}$ ) contained either Triton, filipin, or the appropriate solvent control (dimethylformamide) in $0.68 \mathrm{ml}$ of the isotonic salt mixture. The final Triton concentration (after addition of liposomes) was I\% which was sufficient to produce complete release of the trapped markers; the concentration of filipin was varied as desired and the amount of dimethylformamide never exceeded $5 \%$. The reaction was initiated by the addition of $0.32 \mathrm{ml}$ of the liposome preparation. The final concentration of the liposomes is thus about $2.5 \mathrm{mg} / \mathrm{ml}$ on the basis of lecithin content. This amount was chosen because it corresponded to the concentration of lipid dispersion used in the previous electron microscopic investigation ${ }^{11}$, and, therefore, facilitated comparison of the different experiments. After incubation at $37^{\circ}$ for $30 \mathrm{~min}, 0.7 \mathrm{ml}$ of the reaction mixture was removed and dialysed for $\mathrm{I} h$ with shaking against $\mathrm{I} 5 \mathrm{ml}$ of the isotonic salt mixture at room temperature. Preliminary experiments indicated that incubation for longer than $30 \mathrm{~min}$ did not result in any greater release of marker and that dialysis equilibrium was achieved in $\mathrm{I} h$ under the above conditions. The dialysate was subsequently assayed for either glucose or phosphate. Glucose was determined by the fluorimetric procedure of LowRY et al. ${ }^{12}$ and phosphate was assayed by minor modification of the method used by GERLACH AND DEUTICKE ${ }^{13}$. The amount of marker released by Triton or filipin was corrected for the amount released in control tubes and the results are expressed as the percent of the maximum released by Triton.

\section{Preparation and assay of liposomes: "micro method"}

Chloroform solutions of lecithin (containing 2-3 $\mu$ moles), dicetyl phosphate, and

Biochim. Biophys. Acta, I52 (1968) $\mathrm{I}_{74-185}$ 
cholesterol in the desired molar ratio were added to Io-ml conical flasks. The solvent was removed by a stream of $\mathrm{N}_{2}$ and subsequent evacuation in a dessicator $(0.05 \mathrm{~mm}$ $\mathrm{Hg}$ ) for $\mathrm{I} 5 \mathrm{~min}$. Sufficient marker solution $(0.2-0.3 \mathrm{ml}$ of $0.3 \mathrm{M}$ glucose) was added to the dried lipid film to give a final lecithin concentration of Io $\mu$ moles $/ \mathrm{ml}$, and the lipid mixture was dispersed with a Vortex mixer. After $1.5 \mathrm{~h}$ equilibration at room temperature, with occasional agitation, the liposome preparation was dialyzed for I $\mathrm{h}$ against $\mathrm{I} 5 \mathrm{O} \mathrm{ml}$ of the isotonic salt mixture.

The amount of glucose released by Triton or filipin from liposomes prepared by the above procedure was followed by the change in absorbance at $340 \mathrm{~m} \mu$ in the presence of hexokinase and glucose-6-phosphate dehydrogenase. Cuvettes with a Io$\mathrm{mm}$ light path initially contained the following reagents (in order of addition): 0.28 $\mathrm{ml}$ of Tris buffer (o.I M, pH 8); $0.50 \mathrm{ml}$ of double strength isotonic salt mixture (0.I5 $\mathrm{M} \mathrm{KCl}$ and $0.15 \mathrm{M} \mathrm{NaCl}$, prepared in Tris buffer); o. Io ml of magnesium acetate (0.02 $\mathrm{M}$ ); $0.05 \mathrm{ml}$ of ATP (0.02 M); $0.05 \mathrm{ml}$ of TPN (o.0I M); $5 \mu \mathrm{l}$ each of hexokinase and glucose-6-phosphate dehydrogenase solutions (see below). Control cuvettes were identical to the above except that either TPN or glucose-6-phosphate dehydrogenase was omitted. The reaction was initiated by the addition of $5 \mu \mathrm{l}$ of the appropriate liposome preparation. After a stable change in absorbance had occurred (usually within $3 \mathrm{~min}$.), o.Io $\mathrm{ml}$ of Triton (stock solution: $10 \%$ Triton prepared in Tris buffer) or varying amounts of filipin (stock solution: $\mathrm{x} \mathrm{mg} / \mathrm{ml}$ dimethylformamide) was added. In most experiments, the final concentration of dimethylformamide was less than $2 \%$; at this level, the solvent had very little, if any, effect on the rate of glucose release. After addition of the lytic agent, the reaction was followed until a significant increase in absorbance was no longer observed.

The total amount of glucose present in $5 \mu \mathrm{l}$ of liposomes never exceeded 0.3 $\mu$ mole in our experiments. Conditions of assay were such that essentially complete oxidation of this amount of glucose occurred within I-2 min at room temperature. The chief source of variation in this assay is the activity of each of the enzymes. In practice, we have found that stock solutions of hexokinase $(6.7 \mathrm{mg} / \mathrm{ml})$ and glucose6-phosphate dehydrogenase $(3.4 \mathrm{mg} / \mathrm{ml})$, prepared by diluting I vol. of the suspension supplied by Boehringer with 0.5 vol. of cold distilled water, guaranteed attainment of this rate. Enzymes from other commercial sources were occasionally less active and we would recommend that each preparation be assayed before use.

Additional experimental details are provided in the following section.

\section{RESULTS AND DISCUSSION}

\section{Validation of spectrophotometric assay}

Fig. I is a copy of the absorbance changes at $340 \mathrm{~m} \mu$ (traced by a Sargent Model $\mathrm{MR}$ recorder connected to a Zeiss spectrophotometer) and illustrates the basis for the assay used in this investigation. Addition of liposomes at zero time to experimental and control cuvettes produced an immediate increase in absorbance. The increase observed in the absence of TPN was due to light scatter by the liposomes. After the absorbance had attained constant values, the difference between the experimental and control cuvettes was used to calculate the amount of untrapped glucose present in the liposome preparation.

The total amount of glucose present in the liposome preparation can be deter- 

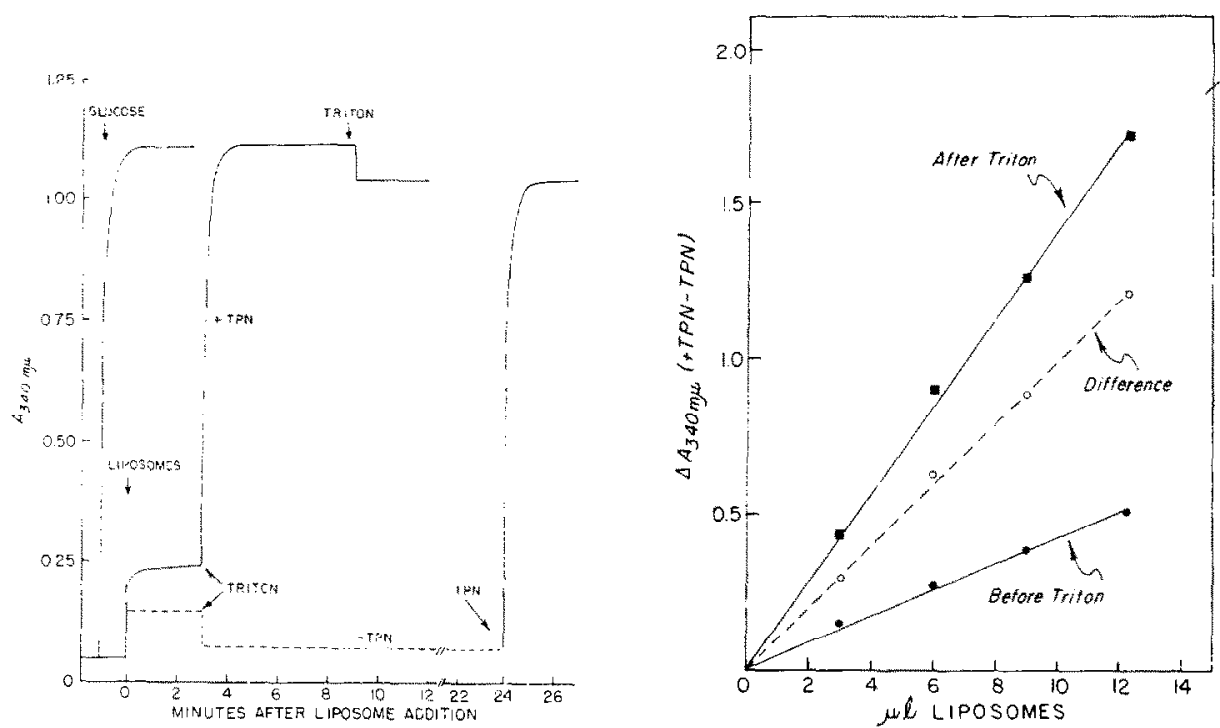

Fig. I. Basis for spectrophotometric assay. Liposomes $(5 \mu 1)$, prepared from egg lecithin, dicetylphosphate, and cholesterol in a molar ratio of $7: 2: 0.5$, were added to experimental $(+T P N)$ and control (-TPN) cuvettes at zero time. Triton $(0.1 \mathrm{ml})$, to give a final concentration of $1 \%$, was added to both cuvettes at $3 \mathrm{~min}$ and the same amount was added to the experimental cuvette again at $9 \mathrm{~min}$. TPN $(0.05 \mathrm{ml})$, to give a final concentration of $0.5 \mu \mathrm{mole} / \mathrm{ml}$ (same as in the experimental cuvette initially), was added to the control cuvette at $24 \mathrm{~min}$. Absorbance values were not corrected for dilution after each addition. The curve on the left was obtained with glucose $(0.17 \mu$ moles $)$ under the above conditions. See text for further details.

Fig. 2. Effect of liposome concentration on the relative amounts of untrapped ("before Triton"). total ("after Triton"), and trapped glucose (by difference). Assay carried out as described in MATERIALS AND METHODS except that the reaction was initiated by addition of varying quantities of liposomes prepared from egg lecithin and dicetyl phosphate in a molar ratio of $7: 2$.

mined from the difference between the experimental and control cuvettes following the addition of sufficient Triton to release the trapped marker. As shown in Fig. I, a rapid rise in absorbance occurred when Triton was added to the experimental cuvette at $3 \mathrm{~min}$; ; in the control cuvette, the absorbance decrcased due to lysis of the liposomes. Complete lysis (i.e. release of all the trapped glucose) had occurred under these conditions because: ( $\mathrm{I}$ ) subsequent addition of another aliquot of Triton to the experimental cuvette at $9 \mathrm{~min}$. did not result in a further increase in absorbance (the apparent decline was due to dilution), and (2) addition of TPN after 24 min. to the control cuvette, containing lysed liposomes, produced a final absorbance value identical to that found in the complete system. Lysis induced by Triton was extremely rapid as indicated by the observation that, when an amount of glucose equivalent to that present in the liposome preparation was assayed under the above conditions, the rate of absorbance increase was indistinguishable from that obtained when the liposomes were treated with the detergent.

The amount of trapped marker contained within the liposome preparation can therefore be calculated from the difference: total minus untrapped glucose. The validity of the present method was further substantiated by the experiment described in Fig. 2 which demonstrates the linear relationship between liposome concentration and the levels of total, untrapped, and trapped glucose. 
Although filipin has an absorption maximum near $340 \mathrm{~m} \mu$, the effect of the antibiotic on the release of glucose from liposomes can also be followed spectrophotometrically provided that the final concentration does not exceed about $\mathrm{I}_{5} \mu \mathrm{g} / \mathrm{ml}$. The applicability of the assay is demonstrated by the experiment described in Fig. 3; on the left, the obscrved absorbance values in the cxpcrimental $(1$ TPN) and control (-TPN) cuvettes are recorded and, on the right, the calculated difference between the experimental and control cuvettes. Liposomes were added at zero time, the reaction allowed to proceed for 5 min until no further change in absorbance was observed, and then filipin was added to both cuvettes. The immediate increase in absorbance was due to the antibiotic per se whereas the subsequent slower increase was due to the
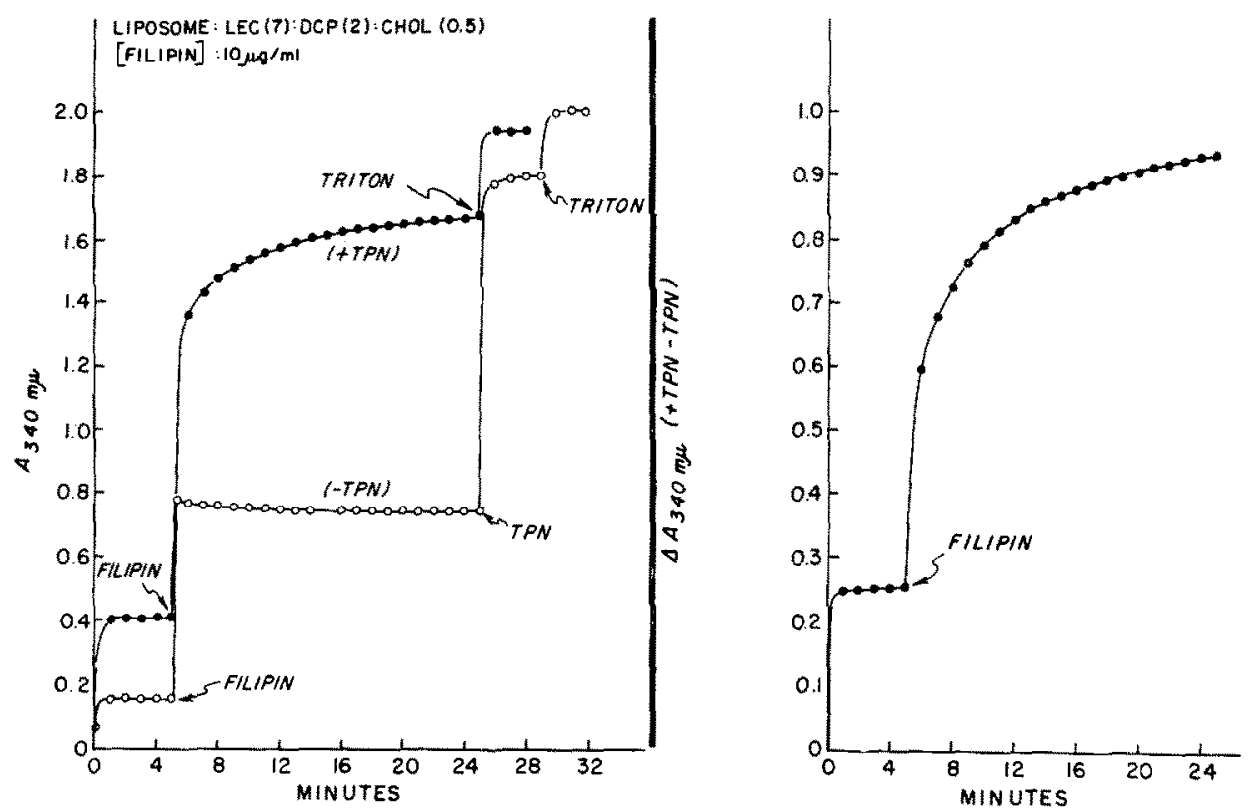

Fig. 3. Spectrophotometric assay lor glucose release from liposomes in the presence of filipin. Liposomes $(5 \mu 1)$, prepared from egg lecithin (LEC), dicetyl phosphate (DCP), and cholesterol (CHOL) in the indicated molar ratios, were added to experimental ( + TPN) and control (-TPN) cuvettes at zero time. Filipin ( $10 \mu \mathrm{l}$ ), to give a final concentration of $10 \mu \mathrm{g} / \mathrm{ml}$, was added to both cuvettes at $5 \mathrm{~min}$; Triton $(0.1 \mathrm{ml})$, to give a final concentration of $1 \%$, was added to the experimental and control cuvettes at 25 and $28 \mathrm{~min}$, respectively; TPN $(0.05 \mathrm{ml})$, to give a final concentration of $0.5 \mu \mathrm{mole} / \mathrm{ml}$ (same as in the experimental cuvette initially), was added to the control cuvette at 25 min. Absorbance values were corrected for dilution following each addition. See text for further details.

release of glucose. The difference between the experimental and control cuvettes at various times after filipin addition is thus a measure of the rate of glucose release (see below). Again, it should be noted that addition of TPN to the control cuvette at 25 min. produced a rapid increase in absorbance due to the glucose which had been released during incubation of the liposomes with filipin. However, unlike the situation described in Fig. I, complete glucose release was not obtained with the concentration of filipin employed in the present experiment because the subsequent addition of Triton caused a further increase in absorbance. 
The spectrophotometric assay obviously can only be used to follow the kinetics of filipin-induced glucose release under conditions in which the antibiotic is involved in the rate-limiting step. The fulfillment of this prerequisite is demonstrated by the experiment described in Fig. 4. As noted in MATERIALS AND METHODS, and as indicated in Fig. I, the concentrations of ATP, TPN, $\mathrm{Mg}^{2+}$, hexokinase, and glucose-6phosphate dehydrogenase present in the cuvettes were such that complete oxidation of the total glucose (trapped plus untrapped) in the liposome preparation occurred within about $\mathrm{I}-2 \mathrm{~min}$. Because the change in $34^{-}-\mathrm{m} \mu$ absorption, which was observed when the liposomes were lysed with Triton at a final concentration of $I \%$, was also completed within this time interval, this assay cannot be employed to follow the kinetics of Triton-induced glucose release. However, in the present investigation, the concentrations of filipin generally used were such that the rate of absorbance increase observed after addition of the antibiotic was significantly less than the rate observed after addition of Triton (Fig. 4). Preliminary experiments failed to reveal any effect of filipin on the activities of either hexokinase or glucose-6-phosphate dehydrogenase which indicates that the enzymes and cofactors were not rate-limiting. Accordingly, in subsequent experiments employing the spectrophotometric assay, the results are expressed as the percent of the maximum glucose release obtained with Triton after correction for the amount of untrapped marker initially present in the liposome preparation.

In contrast to the above results, we were not able to establish that, in the dialysis assay for leakage, filipin was involved in the rate-limiting step. In the experiment

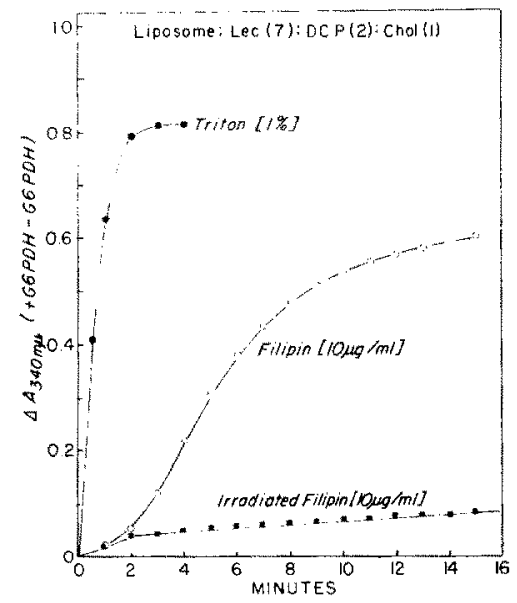

Fig. 4. Rate of glucose release from liposomes in the presence of Triton, filipin, and irradiated filipin. The reaction was initiated by addition of $0.1 \mathrm{ml}$ of Triton, filipin, or the irradiated derivative (see below) to give the final concentrations shown for each curve. The cuvettes already contained liposomes $(5 \mu \mathrm{l})$ prepared from egg lecithin (LEC), dicetyl phosphate (DCP), and cholesterol (Chol) in the molar ratios indicated in the figure. The values recorded are the differences between experimental ( + glucose-6-phosphate dehydrogenase $(\mathrm{G} 6 \mathrm{PDH})$ ) and control (-G6PDH) cuvettes corrected for the initial absorbance difference (due to untrapped glucose) before addition of the lytic agents. See text for further details. A stock solution of irradiated filipin was prepared by illumination of tubes containing $0.90 \mathrm{ml}$ of $\mathrm{NaCl}\left(0.15 \mathrm{M}\right.$ ), o. $0 \mathrm{ml}$ of $\mathrm{FMN}\left(\mathrm{ro}^{-3} \mathrm{M}\right)$, and $10 \mu l$ of filipin (I0 $\mathrm{mg} / \mathrm{ml}$ dimethylformamide) for $30 \mathrm{~min}$ in the apparatus previously described ${ }^{4}$. Control tubes containing only filipin were kept dark. 
described in Fig. 5, liposomes were added to tubes containing the indicated amounts of Triton, antibiotic, or dimethylformamide (solvent control); immediately after mixing, an aliquot $(0.7 \mathrm{ml})$ was removed and dialyzed against $15 \mathrm{ml}$ of the isotonic salt mixture (see MATERIALS AND METHODS). The dialysate was analyzed for glucose at various times after addition of the aliquot to the dialysing sacs. It is apparent that the final equilibrium value of glucose was significantly greater in the incubation mixture containing Triton than in the control, and that the amount of marker released was dependent on the initial antibiotic concentration. When, however, an amount of glucose (in $0.7 \mathrm{ml}$ of the isotonic salt mixture) approximately equivalent to the final equilibrium values was subjected to the same dialysis procedure, it was observed that

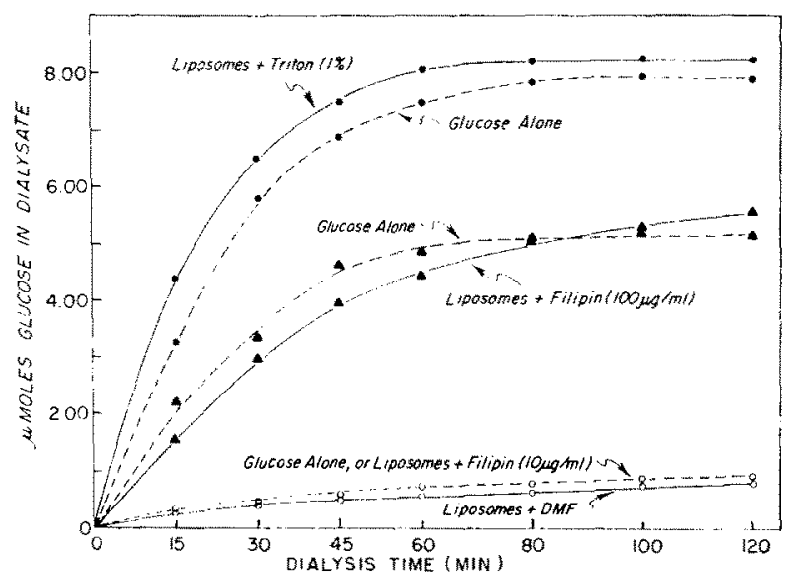

Fig. 5. Rate of glucose appearance in dialysate in the presence of Triton or filipin. Liposomes were prepared by the "macro method" from egg lecithin:dicetyl phosphate (DCP), and cholesterol in a molar ratio of $7: 2: 1$. See text for further details.

the rate of appearance of the marker in the dialysate was essentially identical to that found in samples containing the liposome preparation. Thus, although the dialysis procedure can be conveniently used to determine the total amount of marker released by various lytic agents, it does not appear suitable for following the kinetics of filipin-induced release. The above experiment indicates that, in this assay, the ratelimiting step may involve passage of the marker through the dialysis tubing. These observations are not, necessarily, in contradiction of an analogous experiment described by BANGHAM, STANDISH AND WATkINS ${ }^{1}$ They investigated the release of trapped $\mathrm{K}^{+}$and observed that the diffusion of marker alone through the dialysis sac was several orders of magnitude greater than the leakage of the marker from the liposomes. However, in their experiment, they studied the "endogenous" rate of release from the liposomes in the absence of any lytic agent and the possibility that, in the presence of a lytic agent, the rate of leakage may approach (or exceed) the rate of diffusion across the dialysis membrane, must be considered.

\section{Effect of antibiotic concentration}

The spectrophotometric assay has several advantages. For example, in the preceding experiments, only $5 \mu \mathrm{l}$ of liposome preparation (0.05 $\mu$ mole lecithin) were 
added to each cuvette and appreciable release by filipin was observed with relatively low concentrations of filipin (about $\mathrm{IO}^{-5} \mathrm{M}$ ). The dialysis assay requires more liposomes and higher levels of antibiotic. Thus, in the present investigation, $320 \mu \mathrm{l}(3.2$ $\mu$ moles of liposomal lecithin) and, in the experiments of WEISSMANn AND SESSA, I.o $\mathrm{ml}$ ( $\mathrm{5} 5 \mu$ moles of liposomal lecithin) were employed, and $\mathrm{IO}^{-4}-\mathrm{IO}^{-3} \mathrm{M}$ filipin was necessary to obtain a significant release of marker ${ }^{2}$. The fact that small amounts can be reproducibly assayed is an obvious advantage because it renders feasible the preparation of liposomes from certain natural and synthetic phospholipids which are currently available in limited supply*. Furthermore, use of the spectrophotometric assay made possible the preparation of liposomes on the day of each experiment

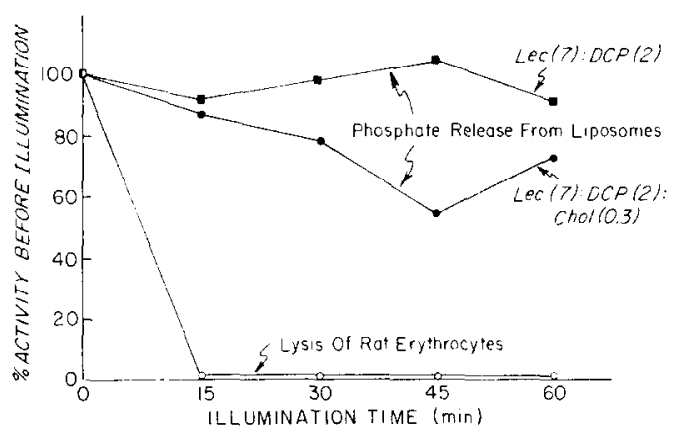

Fig. 6. Effect of illumination time on the ability of filipin to release phosphate from liposomes (dialysis assay) and lyse rat ery throcytes. Phosphate release: Tubes, containing initially $0.57 \mathrm{ml}$ of isotonic salt mixture, o.Io $\mathrm{ml}$ of FMN (10 $\mathrm{O}^{-3} \mathrm{M}$ ), and Io $\mu \mathrm{l}$ of filipin (10 $\mathrm{mg} / \mathrm{ml}$ dimethylformamide) were illuminated in the apparatus previously described ${ }^{14}$ for the times shown on the abscissa. The reaction was then started by addition of $0.32 \mathrm{ml}$ of the appropriate liposomes prepared by the "macro method" from egg lecithin (LEC), dicetyl phosphate (DCP), and cholesterol (Chol) (molar ratios indicated in figure). After incubation for $30 \mathrm{~min}$ at $37^{\circ}, 0.7 \mathrm{ml}$ of the reaction mixture was assayed for the amount of inorganic phosphate released by the dialysis procedure described in MATERIALS AND METHODS. Results are expressed as percent of the phosphate released by IOo $\mu \mathrm{g}$ of non-irradiated filipin (i.e., zero time) after correction for the amount present in tubes containing $1 \%$ dimethylformamide. Erythrocyte lysis: Illumination of $100 \mu \mathrm{g}$ of filipin carried out as described above. The reaction was then started by addition of $0.32 \mathrm{ml}$ of a suspension of washed rat erythrocytes ${ }^{11}$. After incubation for 90 min at $37^{\circ}$, intact cells were removed by centrifugation and the extent of hemolysis was determined spectrophotometrically at $55^{\circ} \mathrm{m} \mu$ by measuring the amount of hemoglobin released into solution. Lysis of the erythrocytes in $\mathrm{I} . \mathrm{o} \mathrm{ml}$ of water gave a supernatant solution with an absorbance of 0.810 ; under the above conditions, complete lysis with non-irradiated filipin (i.e., zero time) occurred within I min following erythrocyte addition.

* Using the dialysis assay, we have previously shown that incorporation of cholesterol into liposomes prepared with egg lecithin reduced the amount of marker (phosphate or glucose) that was released when the liposomes were incubated at various temperatures ${ }^{11}$. These experiments have now been extended with the spectrophotometric assay using a variety of synthetic lecithins ${ }^{10}$. It was observed that cholesterol incorporation reduced the amount of glucose released from liposomes prepared with I-stearoyl,2-oleoyl (18:0/I8:I) and I,2-dioleoyl (I8:I/I8:I) lecithins. The sterol had little effect on the amount of marker released from liposomes prepared with 1,2 -dilinoleoyl (18:2/18:2) phosphatidyl choline. These results seem significant because monolayer studies ${ }^{18}$ have shown that the mean molecular area of $18: 0 / 18: 1$ and $I 8: 1 / 18:$ I phosphatidyl cholines is reduced by cholesterol whereas the sterol has no detectable effect on di-18:2 lecithin. Furthermore, filipin has been shown to interact with monolayers of ergosterol and cetyl alcohol at low molar ratios of antibiotic/lipid 7,9 and these compounds can substitute for cholesterol in "stabilizing" the liposomes. Filipin does not interact with monolayers of cholesterol acetate and sterol esters also do not reduce the amount of glucose rcleased from the liposomes. These observations suggest that the polyene antibiotics may perhaps interfere with the ability of sterols to stabilize the bilayer configuration of phospholipids.

Biochim. Biophys. Acta, I52 (1968) I $74^{-1} 85$ 
(about $3 \mathrm{~h}$ are required) whereas, with previous procedures, prolonged dialysis (about $\mathrm{I}_{5} \mathrm{~h}$ ) was necessary to remove most of the untrapped marker. The present method thus reduces the likelihood that the lecithins may have undergone degradative changes (e.g., hydrolysis, oxidation) during the course of liposome preparation.

From the point of vicw of this investigation, the most significant feature of the spectrophotometric assay is its sensitivity to lower concentrations of filipin. Development of this method was, in fact, prompted by the experiment described in Fig. 6 which demonstrates that use of high concentrations of lytic agents may occasionally produce misleading results. It has been previously shown that illumination of filipin with visible light in the presence of flavin nucleotides abolishes the anti-fungal and hemolytic properties of the antibiotic ${ }^{15}$. When compared with the parent antibiotic, the irradiated derivative produces a smaller increase in the surface pressure of cholesterol monolayers ${ }^{9}$ and has a negligible effect on the electrical resistance of lecithincholesterol bilayers ${ }^{18}$. However, as indicated in Fig. 6, illumination of filipin for as long as $\mathrm{I} h$ has little effect on the ability of the antibiotic to induce the leakage of marker from liposomes when tested at a concentration ( $100 \mu \mathrm{g} / \mathrm{ml}$ ) normally employed in the dialysis assay. At this concentration, the irradiated derivative is, nevertheless, devoid of biological activity after I5-min illumination. In contrast, the irradiated derivative did not cause the leakage of glucose in the spectrophotometric
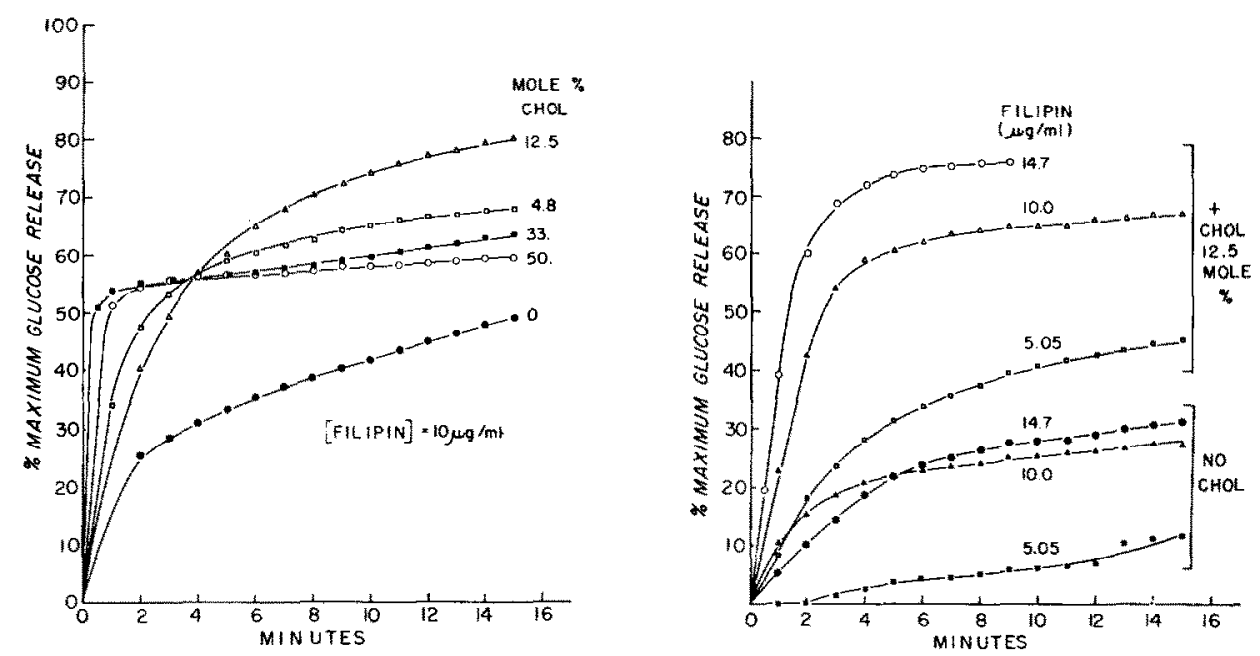

Fig. 7. Effect of cholesterol (CHOL) incorporation into liposomes on the rate and extent of glucose release in the presence of filipin. The reaction was initiated by the addition of ro $\mu$ l of filipin ( $\mathrm{mg} / \mathrm{ml}$ dimethylformamide) to cuvettes which already contained $5 \mu$ l of the appropriate liposome preparation. Liposomes were prepared from egg lecithin and dicetyl phosphate in a molar ratio of $7: 2$, respectively, with varying amounts of cholesterol. In this experiment, and the one described in Fig. 8, the content of dicetylphosphate was not included in the calculation of the "mole \% cholesterol" which is given by the expression: $100 \times$ (moles sterol/moles sterol + moles lecithin). Glucose release was determined by the spectrophotometric assay as described in the text.

Fig. 8. Effect of filipin concentration on the rate and extent of glucose release from liposomes with and without cholesterol (CHOL). Experiment similar to that described in Fig. 7 except that the reaction was initiated by the addition of varying quantities of filipin to give the final concentrations indicated. 
assay (Fig. 4). It is thus possible that the release of marker observed with high concentrations of filipin may be due to factors other than direct interaction with the liposomes (e.g., reduction in the surface tension of water).*

\section{Effect of cholesterol incorporation on the filipin sensitivity of liposomes}

On the basis of the above experiments, the effect of cholesterol incorporation on the response of liposomes to filipin was re-examined with the spectrophotometric assay. Fig. 7 shows that liposomes, prepared with varying amounts of cholesterol, are more sensitive to the antibiotic than liposomes prepared with lecithin alone. Both the initial rate and the total amount of glucose release are greater when cholesterol is present. The reason why the rapid rate, observed with liposomes containing a high mole percent of sterol $(e . g ., 50 \mathrm{~mole} \%$ ), declines markedly after I min is not yet known. It is possible that, under these conditions, not all of the cholesterol is incorporated into the liposome structure; the unincorporated sterol may compete with the liposomes for the available antibiotic. Fig. 8 demonstrates that liposomes, prepared with a lower molar ratio of sterul:lecithin ( $12.5 \mathrm{~mole} \%$ ), are more sensitive to filipin than liposomes from which cholesterol has been omitted, over the concentration range which can be employed in the assay.

WEISSMANN AND SESSA ${ }^{2}$ have earlier demonstrated that incorporation of cholesterol increases the sensitivity of liposomes to amphotericin B and nystatin and we have been able to confirm this observation using the spectrophotometric assay (unpublished experiments)**. These observations, together with the present results, are thus consistent with the view that all polyenes share the same basic mechanism, and that differences in the extent of membrane damage induced by the antibiotics may reflect varying affinities for sterol in the cell membrane $e^{5,9}$. We have previously suggested $^{\theta}$ that the discrepancy between the known selective toxicity of filipin, and the results obtained with liposomes by WeIssmann and SEsSa, may have been due to the high antibiotic concentrations employed in their experiments (see INTRODUCTION). This contention is supported by the current investigation and indicates that results obtained with model membrane systems in the presence of high concentrations of "lytic" agents must be interpreted with caution. This restriction obviously applies not only to liposomes but also to lipid monolayers and bilayers as well. For example, we have found that the polyenes can interact with monolayers ${ }^{\mathbf{9}}$ and bilayers ${ }^{\mathbf{2 0}}$ prepared without sterol at high molar ratios of antibiotic/lipid. These results would lead one to predict that the polyenes should be lethal to organisms devoid of sterol in their cell membrane; to our knowledge, this has not been observed.

* Our electron microscopic observations ${ }^{11}$ support the possibility that the release of marker, which is observed with high concentrations of the filipin, may proceed by different mechanisms depending on whether cholesterol is present. Thus, the antibiotic produced "pits" in lecithincholesterol dispersions whereas the characteristic lamellar pattern was retained when these were treated with the irradiated derivative. Similarly, filipin had no visible effect on dispersions from which sterul was omitted.

** However, Weissmann AND SEssa ${ }^{2}$ were able to obtain only a slight release of marker with etruscomycin (one of the most potent polyene antibiotics) and no release with pimaricin. This has been also our experience to date with these antibiotics using the spectrophotometric assay. Nevertheless, these antibiotics do interact with monolayers ${ }^{9}$ and bilayers ${ }^{20}$ prepared with cholesterol. The reason for this discrepancy between the various membrane models is not yet known.

Biochim. Biophys. Acta, I52(1968) 171-185 


\section{ACKNOWLEDGEMENTS}

Work in the authors' laboratories has been supported by U.S. Public Health Service Grant AI-05II4 and a Career Development Award (to S.C.K.), and funds provided by the Netherlands Organization for the Advancement of Pure Research (to L.L.M.V.D.). J.H. is a recipient of a N.D.E.A. fellowship.

\section{REFERENCES}

I A. D. Bangham, M. Standish and J. C. Watkins, $J$. Mol. Biol., 13 (1965) 238.

2 G. Weissmann and G. Sessa, $J$. Biol. Chem., 242 (1967) 616.

3 G. Sessa and G. Weissmann, Biochim. Biophys. Acta, i35 (1967) 4 r6.

4 J. O. Lampen, Symp. Soc. Gen. Microbiol., I6 (Ig66) I I I.

5 S. C. Kinsky, S. A. Luse and L. L. M. VAN DeEnen, Federation Proc., 25 (1966) 1503.

6 S. C. Kinsky, in D. Gotrlieb and P. D. Shaw, Antibiotics. I. Mechanism of Action, Springer, Berlin 1967 .

7 R. A. Demel, L. L. M. Van Deenen and S. C. Kinsky, J. Biol. Chem., 240 (1965) 2749.

8 H. Van Zutphen, L. L. M. Van DeEnen and S. C. Kinsky, Biochem. Biophys. Res. Commun., 22 (I966) 393 .

9 R. A. Demel, F. J. L. Crombag, L. L. M. Van Deenen and S, C. Kinsky, Biochim. Biophys. Acta, $150(\mathrm{r} 968) \mathrm{r}$.

io G. Weissmann, G. Sessa and S. Weissmann, Biochem. Pharmacol, r 5 (1966) i 537 .

ir S. C. Kinsky, S. A. Luse, D. Zopf, L. L. M. VAN DeEnen and J. HAXBY, Biochim. Biophys. Acta, $135(1967) 844$.

I2 O. H. Lowry, J. V. Passonneau, F, X. Hasselberger and D. W. Schulz, J. Biol. Chem., $239(1964) 18$.

I3 E. Gerlach and B. Deuticke, Biochem. $Z$., 337 (1963) 477.

I 4 S. C. Kunsky, Proc. Natl. Acad. Sci. U.S., 48 (rg62) 1049.

I5 S. C. Kinsky, R. A. Demel and L. L. M. Van Deenen, Biochim. Biophys. Acta, 135 (1967) 835 .

i6 S. C. Kinsky, S. A. Luse, H. Van Zutphen and L. L. M. Van Deenen, Federation Proc, 26 (1967) 862.

17 O. Ceder and R. RYhage, Acta Chom. Scand., 18 (1964) 558.

I8 R. A. Demet, L. L. M. Van Deenen and B. A. Pethica, Biochim. Biophys, Acta, r35 (1967) II.

I9 R. A. Demel, S. C. Kinsky and L. L. M, Van Deenen, in preparation.

$20 \mathrm{H}$. VAN Zutphen, L. L. M. VAN Deenen and S. C. Kinsky, in preparation.

Biochim. Biophys. Acta, $152(1968) \times 74-185$ 